\title{
Dual-rotor misalignment fault quantitative identification based on DBN and improved D-S evidence theory
}

\author{
Yang Dalian ${ }^{1, *}$, Zhang Fanyu ${ }^{1}$, Miao Jingjing ${ }^{1}$, Zhang Hongxian ${ }^{1,3}$, Li Renjie $^{1}$, and Tao Jie $^{2}$ \\ ${ }^{1}$ Hunan Provincial Key Laboratory of Health Maintenance for Mechanical Equipment, Hunan University of Science and \\ Technology, Xiangtan 411201, PR China \\ 2 School Computer Science and Engineering, Hunan University of Science and Technology, Xiangtan 411201, PR China \\ ${ }^{3}$ Lushan College of Guangxi University of Science and Technology, Liuzhou 545006, PR China
}

Received: 30 June 2020 / Accepted: 5 March 2021

\begin{abstract}
Misalignment fault is the main factor that affects the normal running of dual-rotor system. Quantitative identification the misalignment fault is an important way to ensure the safe and stable service of the dual-rotor system, while the identification accuracy of traditional methods is low. Aiming at the above problems, this paper proposed a dual-rotor misalignment fault quantitative identification method based on DBN and D-S evidence theory improved by mutual information measure (MIMD-S). Seven groups experiments were conducted and several vibration signals were collected. By comparing it with the traditional methods D-S, and Pignistic improved D-S (PD-S) evidence theory, the results show that the method proposed in this paper improves the accuracy of the misalignment fault quantitative identification of the dual-rotor, the identification error rate was only $0.36 \%$.
\end{abstract}

Keywords: Deep belief network / mutual information measure / D-S evidence theory / dual-rotor system / misalignment fault quantitative identification

\section{Introduction}

Dual-rotor system is the key component of modern aeroengine. Due to manufacturing and assembling errors, bearing wear and structural deformation, the dual-rotor system is prone to various types of misalignment faults [1], including coupling parallel misalignment, coupling angle misalignment, and dual-rotor misalignment [2]. The misalignment can increase the vibration amplitude of aeroengine end even cause the structural damage of rotor system, which will threaten the safe service of aero-engine [2]. Fortunately, if the misalignment fault parameters (type, degree) of the dual-rotor can be accurately and timely obtained by people, and then maintenance measures can be taken immediately so that the accidents caused by misalignment faults can be reduced. Scholars have done some relevant studies, Lal and Tiwari [3] identified the misalignment quantitatively by finding the relationship between the fundamental frequency amplitude of vibration signal and the misalignment of rotor system. Rybczynski [4] proposed a method to estimate the misalignment

\footnotetext{
* e-mail: hyyd1216@163.com
}

degree by measuring the position and size of the axis track of the bearing and the rotation direction of the rotor. However, most of the previous studies only identify the misalignment fault or carried out a simple qualitative analysis of misalignment. Therefore, the quantitative identification method of misalignment fault needs to be further studied.

Researchers have presented many methods for identification the misalignment faults, including model-based methods [5,6], instrument-based measurement methods [7] and vibration-based methods [8-10]. However, most of the model-based methods use simplified models, which are different from the actual complex structure of the dualrotor system. The instrument-based measurement methods are unavailable because the aero-engine dual-rotor works in a narrow and closed space. Thus, the vibrationbased methods are the most suitable for identification the misalignment faults of aero-engine dual-rotor. Up to now, many vibration-based methods have been proposed, such as Fourier Transform (FFT) [11,12], Wavelet Transform (WT) [13-15], Hilbert Huang Transform (HHT) [16,17], Empirical Mode Decomposition (EMD) [18,19]. Many classifiers such as neural network (NN) [20], support vector machine (SVM) [21], fuzzy logic (FL) [15,22]. However, 
these traditional methods are all "shallow learning" methods, which facing many problems such as dimensional disaster, overfitting or under-learning [23,24].

In 2006, Hinton with his colleagues proposed deep belief network (DBN) [25], which is a neural network with multiple hidden layers. The DBN is trained layer by layer in an unsupervised manner, it can effectively solve the problems of traditional methods, including the difficulty of feature learning and extraction, easy to fall into the local minimum during training. Moreover, the DBN has strong nonlinear processing ability and good discriminant ability, so that it has been widely used in the many fields, such as image processing [26], human action identification [27], natural language processing [28]. The DBN model has been preliminarily applied in bearing fault diagnosis [29-31] and gear fault diagnosis [29,32-34] since it has been applied to aero-engine structural health identification by scholars [34], however, it has not yet been reported for the identification of fault parameters in double-rotor misalignment.

Actually, the dual-rotor is located in a tightly sealed internal space and work under high temperature and high speed conditions. The sensor cannot be directly mounted on the rotor but only the external casing. Due to the vibration signal is interfered by strong noise when transmitted from the misaligned excitation source to the external measurement location, so that the misalignment fault information obtained by the sensor is very weak. Thereby, only using a sensor to collect the signal cannot accurately identify the misalignment fault parameters. It is necessary to use multiple sensors to collect vibration signals synchronously and fuse the collected signals. The information fusion methods are divided into signal fusion [35], feature fusion [36] and decision fusion [37]. Decision fusion is the highest level of information fusion, because it has the advantages of strong anti-interference, high flexibility and good fault tolerance. Common fusion decision methods include D-S (Dempster-Shafer) evidence theory [38,39], Bayesian decision [40,41], fuzzy logic [42]. The D-S evidence theory is the most commonly used method which was first proposed by Dempster in 1967, and it has been applied in many fields, such as multi-attribute decision-making, information fusion, and intelligence analysis [43]. The D-S evidence theory has a positive application prospect, but due to the conflict between evidence bodies, the D-S decision rule may give contradictory results to the actual solutions, and it will appear the paradox phenomenon [44]. In order to solve the conflict between evidence bodies, scholars have used the distance measure to represent the evidences $[45,46]$. However, the traditional distance measure cannot completely avoid the conflict in some cases. Mutual information is a measure of the interdependence of variables in information analysis filed, and it has been applied as a similarity measure indicator $[47,48]$. However, there have been no reports on its application to resolve conflicts of the D-S evidence, the main reason is that the construction method based on the mutual information to express the conflict between the evidence bodies is still unclear, which needs to be studied more deeply.

To sum up, this paper focuses on the dual-rotor system consisting of an outer rotor and an inner rotor. To solve the problem of low recognition rate of traditional methods due to the complexity and diversity of misalignment faults, This paper studies the diagnosis method based on DBN and D-S evidence theory. Firstly, $N$ DBN models are trained with vibration signals collected by $N$ sensors; secondly, the identification results of $N$ DBN models are used as the original evidence source, and the D-S evidence combination rule is improved based on the mutual information distance; finally, the revised evidence sources are fused in order to obtain the diagnosis results.

\section{D-S evidence theory}

Firstly, the D-S evidence theory divides the problem to be analyzed into several sub-problems, and then solves each sub-problem respectively, finally, Dempster's rule is adopted to fuse the multiple information. Its basic modules include identification framework, basic probability assignment function and combination rules.

The evidence completes the reasoning based on a finite set space that is mutually exclusive and non-empty. Suppose that the set of all possible solutions of the problem to be decided is represented as $\Theta$, in which any two elements are mutually exclusive, therefore, the decision result of the problem must be one of the elements, then the set is called the recognition framework, that is,

$$
\Theta=\left\{A_{1}, A_{2}, \ldots A_{i}, \ldots, A_{n}\right\}
$$

where, $A_{i}$ is the $j$ th element of the identification frame $\boldsymbol{\Theta}$, $n$ is the number of elements, $i=1,2, \ldots, \mathrm{n}$.

The power set of the recognition framework is defined as $2^{\Theta}$, and its basic probability assignment function is defined as $m(\cdot)$, then the following conditions are satisfied:

$$
\left\{\begin{array}{l}
0 \leq m(A) \leq 1 \\
m(\emptyset)=0 \\
\sum_{A \subseteq \Theta} m(A)=1
\end{array}\right.
$$

where, $m(A)$ is the basic probability assignment value of $A$, which indicates the degree of evidence's trust in $A$. The symbol $\emptyset$ represents an empty set, and $m(\emptyset)=0$ indicates that the trust is 0 for the empty set.

For the same problem, if the sources of information are different, several different basic probability assignment functions will be obtained. Therefore, these different basic probability assignment functions need to be fused into a basic probability assignment function. The basic fusion strategy of D-S evidence theory is to calculate the orthogonal sums of these different basic probability assignment functions, which can be expressed as $\oplus$. The D-S evidence combination rules for the two evidence bodies $\left(E_{1}, E_{2}\right)$ are defined as follows:

$$
\left\{\begin{array}{l}
\sum_{1 \oplus 2}(C)=\frac{\forall A, B \subseteq \Theta \mid A \cap B}{1-k} m_{1}(A) m_{2}(B) \\
m_{1 \oplus 2}(C)=0 \quad, C=\emptyset \\
k=\sum_{A \cap B=\emptyset} m_{1}(A) m_{2}(B)
\end{array}\right.
$$


among them, $k$ is the conflict coefficient between the evidence bodies, $m_{1}(\cdot)$ is the basic probability assignment function of the evidence bodies $E_{1}, m_{2}(\cdot)$ is the basic probability assignment function of the evidence bodies $E_{2}$, $m_{1 \oplus 2}(\cdot)$ is the basic probability assignment function of the fused evidence bodies $E_{1 \oplus 2}, A$ is the focal element of the evidence bodies $E_{1}$, and $B$ is the focal element of the evidence body $E_{2}, C$ is the focal element of the evidence body $E_{1 \oplus 2}$. The D-S fusion rule also has the properties of exchange law and binding law.

The exchange law is expressed as:

$$
m_{1} \oplus m_{2}=m_{2} \oplus m_{1}
$$

The binding law is expressed as:

$$
m_{1} \oplus m_{2} \oplus m_{3}=\left(m_{1} \oplus m_{2}\right) \oplus m_{3}=m_{1} \oplus\left(m_{2} \oplus m_{3}\right)
$$

where, $m_{1}, m_{2}$ and $m_{3}$ are the basic probability assignment functions under the same identification condition $\Theta$.

\section{Misalignment faults identification algorithm construction}

\subsection{Mutual information measure D-S evidence theory}

When the D-S evidence theory is used to make the fusion decision for the multi-source information of the misalignment fault of the double-rotor system, the construction method of the recognition framework determines that each result obtained from a single evidence body. Therefore, if there is a big difference between the decision results, there will be serious evidence conflict, which will result in the D-S fusion rule not being used or drawing a conclusion that are contrary to the real solution.

Conflicts between evidence bodies can be expressed using distance. The higher the distance between evidence bodies, the greater the conflict. Therefore, analyzing the paradox of evidence and measuring the similarity between the evidence bodies are the key to resolving the evidence conflicts.

In this paper, the D-S evidence theory fusion method is improved by mutual information measure (MIM), and the mutual information function is defined as follows: suppose recognition framework $\Theta$ has $n$ evidence bodies, which are named as: $E_{1}, E_{2}, \ldots, E_{n}$, the mutual information measure value $D_{m_{i} m_{j}}$ is expressed as:

$$
D_{m_{i} m_{j}}=1-M I_{m_{i} m_{j}}
$$

where, $M I_{m_{i} m_{j}}$ is the mutual information amount of the two evidence bodies, $m_{i}$ and $m_{j}$ respectively represent the basic probability assignment functions. Additionally, it is satisfies: $D_{m_{i} m_{j}}=D_{m_{j} m_{i}}, 0 \leq D_{m_{i} m_{j}} \leq 1,1 \leq i \leq n, 1 \leq j \leq n$.

The steps of the improved evidence fusion method based on MIM proposed in this paper are as follows:

- Calculate the MIM $D_{m_{i} m_{j}}$ between the evidence bodies use the formula (6).
- Establish a similarity measure function between evidence bodies as follows:

$$
S I\left(m_{i}, m_{j}\right)=1-D_{m_{i} m_{j}}
$$

Then, the similarity degree matrix $S I$ is defined as:

$$
S I=\left[\begin{array}{cccc}
1 & 1-D_{m_{1} m_{2}} & \ldots & 1-D_{m_{1} m_{n}} \\
1-D_{m_{2} m_{1}} & 1 & \ldots & 1-D_{m_{2} m_{n}} \\
\vdots & \vdots & \vdots & \vdots \\
1-D_{m_{n} m_{1}} & 1-D_{m_{n} m_{2}} & \ldots & 1
\end{array}\right]
$$

- The total similarity $S I^{\prime}\left(m_{i}\right)$ of the evidence body $E_{i}$ can be obtained by adding each row in the similarity degree matrix SI:

$$
S I^{\prime}\left(m_{i}\right)=\sum_{j=1, j \neq i}^{n} S I\left(m_{i}, m_{j}\right)
$$

It can be known from formula (9) that the larger $S I^{\prime}\left(m_{i}\right)$ is, the larger the total similarity is.

- Calculate the credible function $C\left(m_{i}\right)$ of the evidence body:

$$
C\left(m_{i}\right)=\frac{S I^{\prime}\left(m_{i}\right)}{\max _{1 \leq j \leq n} S I^{\prime}\left(m_{j}\right)}
$$

where, $\forall i, j \in[1, n]$.

- Revise the original evidence source. Formula (20) is used to obtain the credibility of each evidence body and take it as the weight to the original evidence source, that is $\alpha_{i}=C\left(m_{i}\right)$, there are:

$$
\left\{\begin{array}{l}
{m^{\prime}}_{i}(A)=\alpha_{i} m_{i}(A), \forall A \subset \Theta, A \neq \Theta \\
m_{i}^{\prime}(\Theta)=\alpha_{i} m_{i}(\Theta)+\left(1-m_{i}\right), A=\Theta
\end{array}\right.
$$

where, $\forall i, j \in[1, n], m_{i}^{\prime}(A)$ represents the new basic probability assignment value of the revised focal element, and $m_{i}^{\prime}(\boldsymbol{\Theta})$ represents the uncertainty of the $i$ th evidence after being revised.

- The revised D-S evidence sources were fused by the formula (3).

\subsection{Misalignment faults identification based on DBN and MIMD-S evidence theory}

The DBN model is a multi-layer perceptron neural network consisting of multiple restricted Boltzmann machines (RBMs) [49]. The training process of DBN is divided into two steps. The first step is an unsupervised layer-by-layer greedy learning process. In this step, the output of the lower-level RBM is used as the input of the higher-level RBM, which is transmitted layer by layer in order to form a more abstract and higher-level layer. In the second step, 


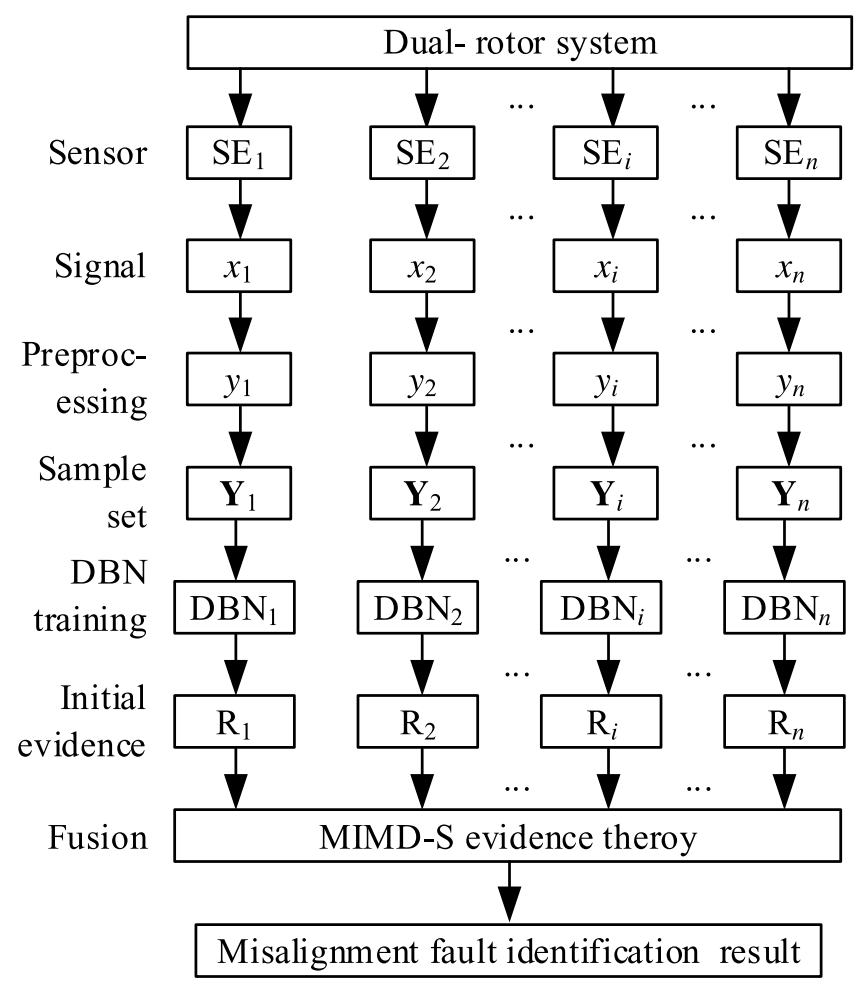

Fig. 1. The misalignment fault identification algorithm diagram based on the DBNMIND-S evidence theory.

the supervised reverse fine-tuning is used to optimize the fault identification capability of the DBN. Secondly, the MIMD-S evidence theory is used to fuse all evidences in order to obtain the fusion diagnosis result. The misalignment faults identification model based on DBN and MIMD-S evidence theory (DBNMIMD-S) is shown in Figure 1, and the specific steps are as follows:

- Define the acceleration vibration signal collected by the $i$ th acceleration sensor $\mathrm{S}_{i}$ as $x_{j}^{i}, i \in(1,2, \ldots, n)$, $j \in(1,2, \ldots, l)$. The vibration signal $x_{i}$ is denoised by a filter to obtain $y_{i}$. From the sensor $\mathrm{S}_{i}, \mathrm{~m}$ groups of data samples are composed of $Y^{i}=\left\lfloor y_{1 j}^{i}, y_{2 j}^{i}, \ldots, y_{m j}^{i}\right\rfloor$.

- The data sample $Y^{i}$ is randomly divided into a training group $Q_{1}^{i}$ and a test group $Q_{2}^{i}$. Taking the $Q_{1}^{i}$ as the input sample of the $\mathrm{DBN}_{i}$, and initialize the layers number, as well as the nodes number of each layer, the iterations number of each RBM, the learning rate, momentum, and other parameters. Finish the greedy training process layer by layer from the low level to the high level, and then gradually and reversely fine-tune the parameters from the highest level to the lowest level according to the label of the training group and the classification rules of the soft-max classifier. Finally, the model $\mathrm{DBN}_{i}$ is obtained, where, $i \in(1,2, \ldots, n)$.

- The test group $Q_{2}^{i}$ is input into the $\mathrm{DBN}_{i}$ model, and the $i$ th preliminary fault identification result is obtained. The $i$ th preliminary identification result is used as the $i$ th original evidence source $\mathrm{R}_{i}$ of the $\mathrm{D}-\mathrm{S}$ evidence theory, where, $i \in(1,2, \ldots, n)$.

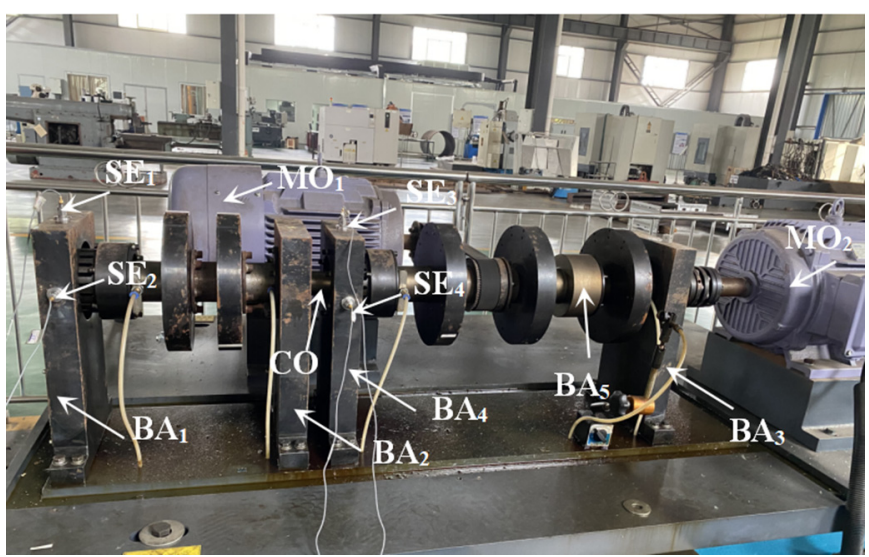

Fig. 2. The experimental rig.

- Finally, the original evidence source is revised by the improved evidence combination method based on mutual information measure, and the revised evidence source is combined by the classic D-S combination rule. Finally, the fault identification result of multi-source decision fusion is obtained.

\section{Misalignment experiments}

\subsection{Experimental setup and data collection}

In order to verify the feasibility and effectiveness of the DBNMIND-S method, a series of dual-rotor misalignment simulation experiments were carried out. These experiments were performed on a dual-rotor vibration test rig which was produced by Suzhou Dongling Vibration Test Instrument co. LTD. of China, Denmark Brüel \& Kjær vibration test system was used to collect vibration signals. The rotor of this test rig is a concentric nested dual-rotor with an inner rotor and an outer rotor. The dual-rotor are supported by five fulcrums, that is, the inner rotor adopts the 1-1-1 support form and the outer rotor adopts the 1-0-1 support form. The two rotors are driven by two servo motors respectively, and the maximum speed is $8000 \mathrm{r} / \mathrm{min}$. Four accelerometer sensors were installed to collect the signal. The experimental rig is shown in Figure 2, where, $\mathrm{SE}_{1}, \mathrm{SE}_{2}, \mathrm{SE}_{3}, \mathrm{SE}_{4}$ are accelerometers; $\mathrm{RO}_{1}$ represents the inner rotor; $\mathrm{RO}_{2}$ represents the outer rotor; $\mathrm{BA}_{1}, \mathrm{BA}_{2}, \mathrm{BA}_{3}$ are the inner rotor support seat; $\mathrm{BA}_{4}, \mathrm{BA}_{5}$ are the outer rotor support seat; $\mathrm{CO}$ is the coupling; $\mathrm{MO}_{1}$ is the outer rotor drive motor; $\mathrm{MO}_{2}$ is the outer rotor drive motor.

In this experiment, the rotor misalignment faults were simulated by adding shims with different thicknesses to different support seats of the dual-rotor vibration test bench. The schematic diagram of the rotor structure and misalignment faults type are shown in Figures $3-5$.

The experiments were divided into seven groups, named as $T_{1}$ to $T_{7}$, the detail conditions are shown in Table 1. In each group of experiments, the speed of the inner rotor was $1500 \mathrm{r} / \mathrm{min}$, and the speed of the outer rotor was $2400 \mathrm{r} / \mathrm{min}$. The rotation directions of the two rotors 
were opposite. The sampling frequency was $16384 \mathrm{~Hz}$. In each group of experiments, several vibration signals were collected.

\subsection{Fusion performance analysis}

A sample set $Y_{j}^{i}$ with dimension of $400 \times 1024$ was obtained from sensor $\mathrm{SE}_{i}$ at experiment $\mathrm{T} j, i \in(1,2,3,4)$, $j \in(1,2, \ldots, 7)$. All sample sets were normalized by the mean-

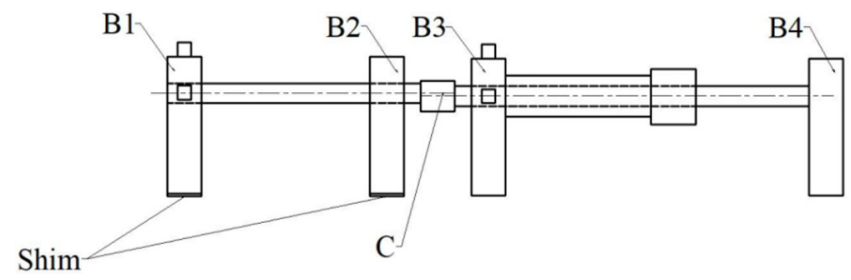

Fig. 3. The schematic diagram of parallel misalignment of coupling.

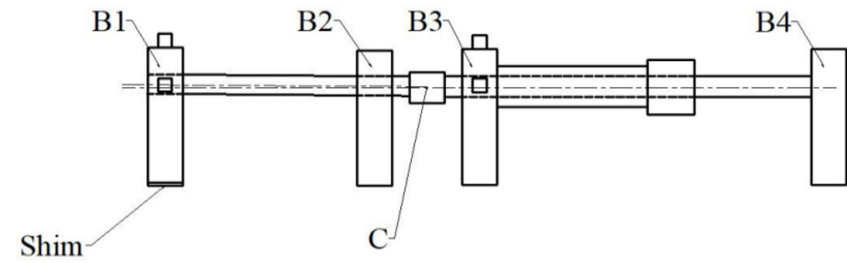

Fig. 4. The schematic diagram of coupling angle misalignment.

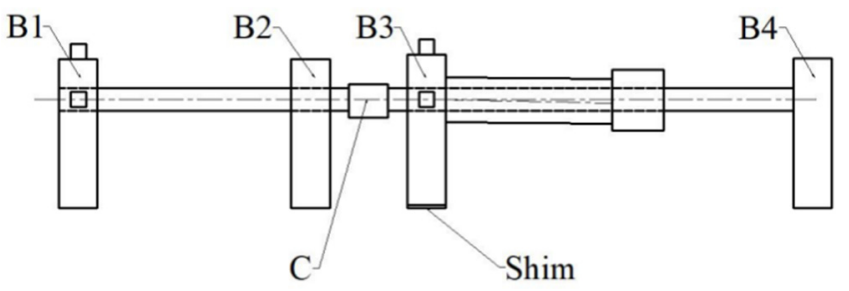

Fig. 5. The schematic diagram of inner and outer rotors misalignment.

Table 1. Description of experimental conditions.

\begin{tabular}{llllll}
\hline Experiment no. & Misalignment faults & \multicolumn{4}{c}{ The thicknesses of gaskets $/ \mathrm{mm}$} \\
\cline { 3 - 6 } & & $\mathrm{B}_{1}$ & $\mathrm{~B}_{2}$ & $\mathrm{~B}_{3}$ & $\mathrm{~B}_{4}$ \\
\hline $\mathrm{T}_{1}$ & Normal & 0.0 & 0.0 & 0.0 & 0.0 \\
$\mathrm{~T}_{2}$ & Coupling parallel misalignment & 0.2 & 0.2 & 0.0 & 0.0 \\
$\mathrm{~T}_{3}$ & Coupling parallel misalignment & 0.5 & 0.5 & 0.0 & 0.0 \\
$\mathrm{~T}_{4}$ & Coupling angle misalignment & 0.2 & 0.0 & 0.0 & 0.0 \\
$\mathrm{~T}_{5}$ & Coupling angle misalignment & 0.5 & 0.0 & 0.0 & 0.0 \\
$\mathrm{~T}_{6}$ & Misalignment of inner and outer rotors & 0.0 & 0.0 & 0.2 & 0.0 \\
$\mathrm{~T}_{7}$ & Misalignment of inner and outer rotors & 0.0 & 0.0 & 0.5 & 0.0 \\
\hline
\end{tabular}

variance method. Randomly select 320 samples from $Y_{j}^{i}$ as training sample set for $\mathrm{DBN}_{i}$, and the rest of samples as the test sample set. Use the training samples to train $\mathrm{DBN}_{1}, \mathrm{DBN}_{2}, \mathrm{DBN}_{3}$, and $\mathrm{DBN}_{4}$. The details information of sample sets for DBNs are listed in Table 2.

5 layers was used in each DBN model, including 1 input layer, 3 hidden layers and 1 classification layer. The number of nodes in the corresponding layer was 1024, 800, 800,1000 , and 7 , respectively. The maximum iteration number of the RBM was 100 , the learning rate was 0.01 , and the momentum was 0.6 . The preliminary identification results of the first 100 test samples are shown in Figure 6.

It can be seen from Figure 6 that there are some errors in the identification results of the DBN model. The model $\mathrm{DBN}_{1}$ gives wrong prediction labels for the test samples numbered 2, 23, 38, 85 and 88 , in Figure $6 \mathrm{a}$. The $\mathrm{DBN}_{2}$ gives wrong prediction labels for the test samples numbered 15,23 , and 38 , in Figure $6 \mathrm{~b}$. The $\mathrm{DBN}_{3}$ gives wrong prediction labels for the test samples numbered 2 only, in Figure 6c. The $\mathrm{DBN}_{4}$ gives wrong prediction labels for the test samples numbered 2, 48, 55, 85 and 98, in Figure 6d. The above results show that different results obtained by different DBN models for same sample, which indicates that the different data collected by different sensors have different effects on different DBN models. Therefore, the identification results of the DBN model trained with data collected by a single sensor are not reliable.

In order to further analyze the differences of different DBN model trained by different sensor signal data, each DBN model was trained 100 times, and the average identification results as listed in Table 3. It can be seen from Table 3 , the $\mathrm{DBN}_{3}$ model has the lowest average error rate, was $3.46 \%$, while the $\mathrm{DBN}_{2}$ model has the highest average error rate, was $4.28 \%$, the identification results of the two models differ by $0.82 \%$. It indicates that the location of the sensor has effects on the identification results and it is difficult to accurately recognize the misalignment fault parameters of aero-engine dual-rotor system by only a sensor. In order to improve the accuracy of identification, multi-sensor fusion decision method must be used for diagnosis.

The multi-source fusion decision method based on DBN and MIMD-S evidence theory was used to recognize the misalignment faults of the dual-rotor system. Firstly, taken the identification results of the DBN models as the original evidence source of the MIMD-S evidence theory and the 
Table 2. The Sample Sets for DBNs.

\begin{tabular}{|c|c|c|c|c|c|}
\hline $\begin{array}{l}\text { Model } \\
\text { no. }\end{array}$ & $\begin{array}{l}\text { Signal } \\
\text { source }\end{array}$ & $\begin{array}{l}\text { Experiment } \\
\text { no. }\end{array}$ & Label & $\begin{array}{l}\text { Dimension of training } \\
\text { sample sets }\end{array}$ & $\begin{array}{l}\text { Dimension of test } \\
\text { sample sets }\end{array}$ \\
\hline \multirow{7}{*}{$\mathrm{DBN}_{1}$} & \multirow{7}{*}{$\mathrm{SE}_{1}$} & $\mathrm{~T}_{1}$ & 1 & \multirow{7}{*}{$2240 \times 1024$} & \multirow{7}{*}{$560 \times 1024$} \\
\hline & & $\mathrm{T}_{2}$ & 2 & & \\
\hline & & $\mathrm{T}_{3}$ & 3 & & \\
\hline & & $\mathrm{T}_{4}$ & 4 & & \\
\hline & & $\mathrm{T}_{5}$ & 5 & & \\
\hline & & $\mathrm{T}_{6}$ & 6 & & \\
\hline & & $\mathrm{T}_{7}$ & 7 & & \\
\hline \multirow{7}{*}{$\mathrm{DBN}_{2}$} & \multirow{7}{*}{$\mathrm{SE}_{2}$} & $\mathrm{~T}_{1}$ & 1 & \multirow{7}{*}{$2240 \times 1024$} & \multirow{7}{*}{$560 \times 1024$} \\
\hline & & $\mathrm{T}_{2}$ & 2 & & \\
\hline & & $\mathrm{T}_{3}$ & 3 & & \\
\hline & & $\mathrm{T}_{4}$ & 4 & & \\
\hline & & $\mathrm{T}_{5}$ & 5 & & \\
\hline & & $\mathrm{T}_{6}$ & 6 & & \\
\hline & & $\mathrm{T}_{7}$ & 7 & & \\
\hline \multirow{7}{*}{$\mathrm{DBN}_{3}$} & \multirow{7}{*}{$\mathrm{SE}_{3}$} & $\mathrm{~T}_{1}$ & 1 & \multirow{7}{*}{$2240 \times 1024$} & \multirow{7}{*}{$560 \times 1024$} \\
\hline & & $\mathrm{T}_{2}$ & 2 & & \\
\hline & & $\mathrm{T}_{3}$ & 3 & & \\
\hline & & $\mathrm{T}_{4}$ & 4 & & \\
\hline & & $\mathrm{T}_{5}$ & 5 & & \\
\hline & & $\mathrm{T}_{6}$ & 6 & & \\
\hline & & $\mathrm{T}_{7}$ & 7 & & \\
\hline \multirow{7}{*}{$\mathrm{DBN}_{4}$} & \multirow{7}{*}{$\mathrm{SE}_{4}$} & $\mathrm{~T}_{1}$ & 1 & \multirow{7}{*}{$2240 \times 1024$} & \multirow{7}{*}{$560 \times 1024$} \\
\hline & & $\mathrm{T}_{2}$ & 2 & & \\
\hline & & $\mathrm{T}_{3}$ & 3 & & \\
\hline & & $\mathrm{T}_{4}$ & 4 & & \\
\hline & & $\mathrm{T}_{5}$ & 5 & & \\
\hline & & $\mathrm{T}_{6}$ & 6 & & \\
\hline & & $\mathrm{T}_{7}$ & 7 & & \\
\hline
\end{tabular}

corresponding DBN models were marked as $\mathrm{E}_{1}, \mathrm{E}_{2}, \mathrm{E}_{3}, \mathrm{E}_{4}$, respectively. The average error rate of test samples of all DBN models in Table 3 were taken as the original uncertainty of the evidence body, that was to say, the uncertainty of $\mathrm{E}_{1}$ was $3.74 \%, \mathrm{E}_{2}$ was $4.28 \%, \mathrm{E}_{3}$ was $3.46 \%$, and $\mathrm{E}_{4}$ was $4.11 \%$. Secondly, the evidence bodies were revised based on the mutual information measure. Finally, the revised evidence bodies were fused according the fusion rules of D-S evidence theory.

In order to analyze the identification performance of the method in this paper, take the fusion records of test samples in Figure 6a-d that are simultaneously recognized wrong by two or more DBN models as examples (No. 2, No. 38 and No. 85). The fusion records are listed in Table 4.

As can be seen from Table 4, the basic probability assignment value of $\mathrm{M}(\mathrm{A} 5)$ in evidence $\mathrm{E}_{1}$ of test sample No. 2 was 0.9626 , which was the maximum value in the identification framework. Therefore, the identification label of $E_{1}$ was 5. Similarly, the identification label of $E_{2}$ was 4 , the identification label of $\mathrm{E}_{3}$ was 1 , the identification label of $E_{4}$ was 3 . Where the results of $E_{1}, E_{3}$ and $E_{4}$ were wrong, only $\mathrm{E}_{1}$ was correctly recognized. It indicates that there was conflicting information in evidence bodies of test sample No. 2, which leaded to different decisions. For the test sample No. 38 and No. 85, there were also conflicting evidence bodies.

This paper used the multi-sensor fusion decision method based on the DBNMIMD-S evidence theory to recognized the three test samples, respectively. It can be seen that after fusion, the maximum probability distribution value of $\mathrm{M}\left(\mathrm{A}_{4}\right)$ in the identification framework of test sample No. 2 was 0.7191 As a matter of course, the identification result of label 4 was correct. Similarly, the maximum value of $\mathrm{M}$ (A6) in the identification framework of test data No. 38 after fusion was 0.5264 , and the identification label 6 was correct. The maximum value of $\mathrm{M}$ (A4) in the identification framework of test data No. 85 after fusion was 0.4988 , and the identification label 4 was also correct. The reason 


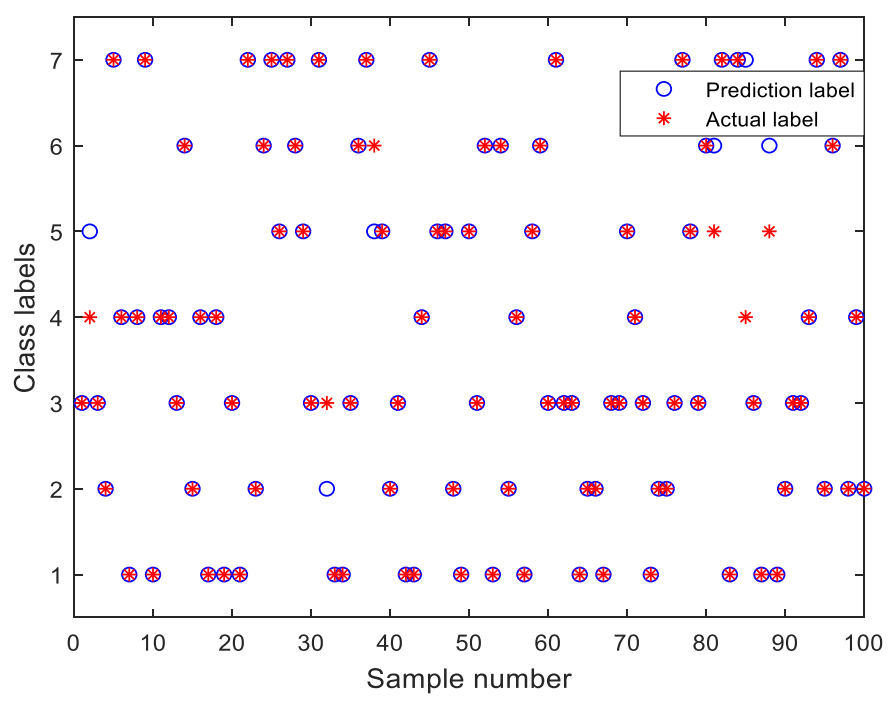

(a)

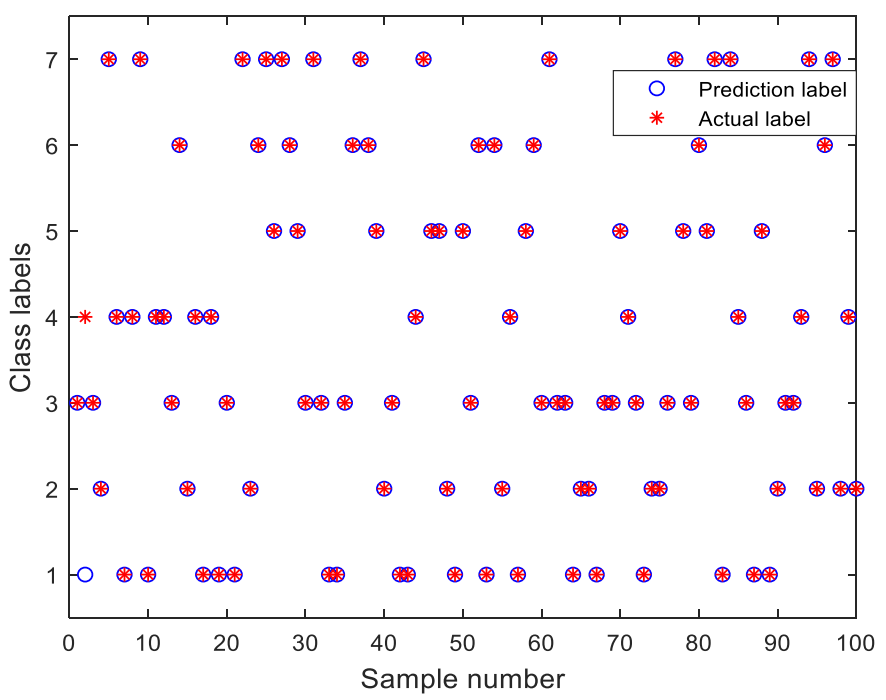

(c)

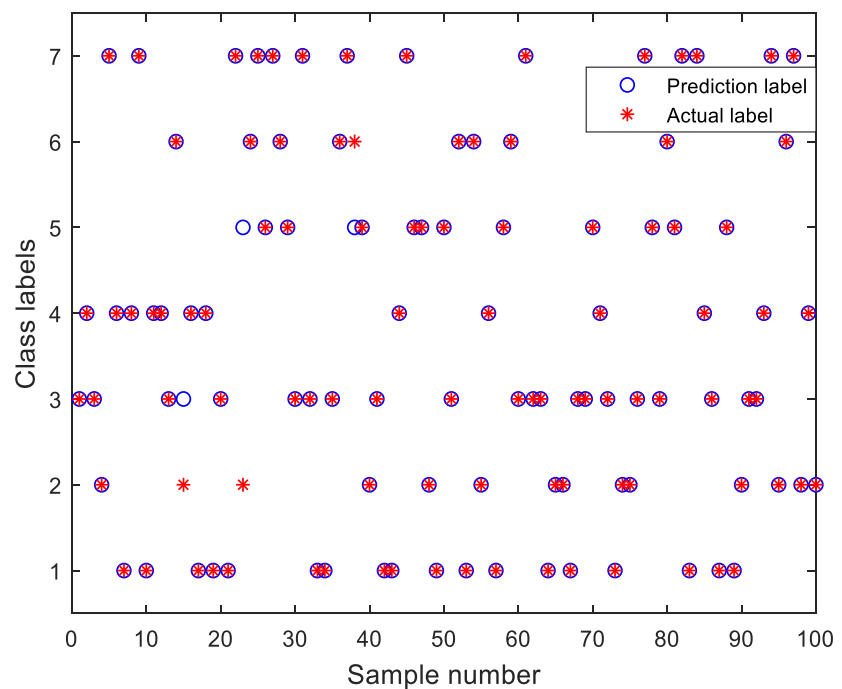

(b)

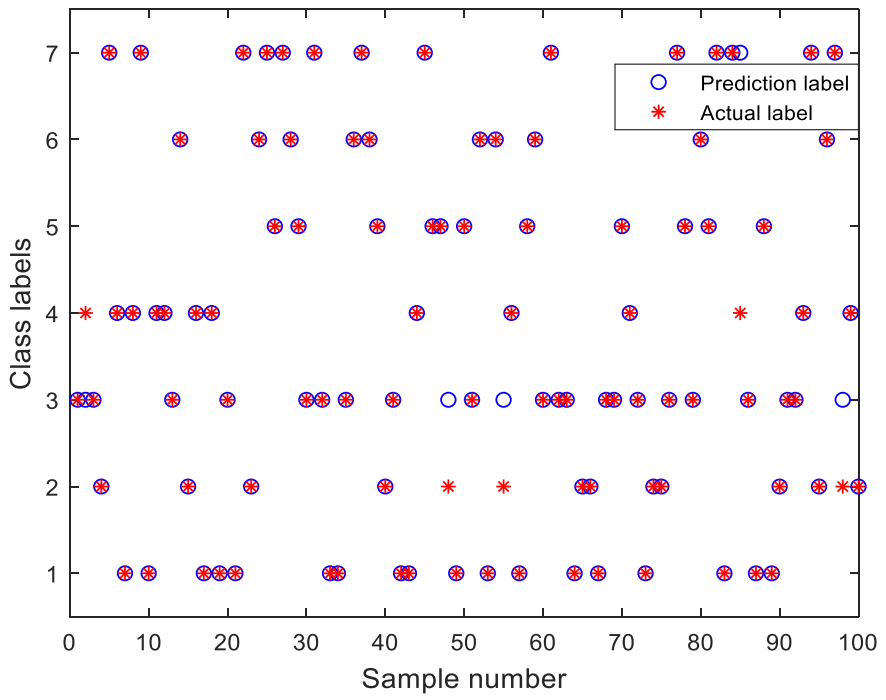

(d)

Fig. 6. Preliminary identification result. (a) $\mathrm{DBN}_{1}$, (b) $\mathrm{DBN}_{2}$, (c) $\mathrm{DBN}_{3}$, (d) $\mathrm{DBN}_{4}$.

Table 3. Error rate of preliminary fault identification.

\begin{tabular}{ll}
\hline Classifier & Average error rate \\
\hline $\mathrm{DBN}_{1}$ & $3.74 \%$ \\
$\mathrm{DBN}_{2}$ & $4.28 \%$ \\
$\mathrm{DBN}_{3}$ & $3.46 \%$ \\
$\mathrm{DBN}_{4}$ & $4.11 \%$ \\
\hline
\end{tabular}

is that the original evidence sources were corrected by weighting the similarity degree, which solved the problem of conflict between evidence bodies effectively so as to recognized the misalignment faults parameters of dual-rotor system accurately.

\subsection{Results and analysis}

In order to verify the effectiveness of the method proposed in this paper, two traditional fusion methods: D-S and Pignistic improved D-S (PD-S), were also selected for comparison. The identification results are shown in Table 5 .

It can be seen from Tables 3 and 5 , the error rates of dual-rotor misalignment faults parameters identification use the multi-sensor fusion decision method is obviously lower than that of dual-rotor misalignment faults identification use a single sensor. This is because the D-S evidence theory fusion decision method can deal with the uncertainty caused by the randomness and fuzziness of information. The D-S evidence theory uses the evidence to represent the uncertainty of information, and uses the fusion rule to deal with the uncertain information, so it can obtain the best result from insufficient and inaccurate information. 
Table 4. Fusion record.

\begin{tabular}{|c|c|c|c|c|c|c|c|c|c|c|c|}
\hline $\begin{array}{l}\text { Sample } \\
\text { no. }\end{array}$ & $\begin{array}{l}\text { Sample } \\
\text { label }\end{array}$ & Evidence & \multicolumn{8}{|c|}{ The basic probability assignment value } & $\begin{array}{l}\text { Recognition } \\
\text { label }\end{array}$ \\
\hline \multirow[b]{2}{*}{2} & \multirow[b]{2}{*}{4} & $\mathrm{E}_{2}$ & 0.0000 & 0.0000 & 0.0000 & 0.9572 & 0.0000 & 0.0000 & 0.0000 & 0.0428 & 4 \\
\hline & & $\mathrm{E}_{3}$ & 0.7975 & 0.0031 & 0.0000 & 0.1643 & 0.0000 & 0.0001 & 0.0004 & 0.0346 & 1 \\
\hline \multirow{5}{*}{38} & \multirow{5}{*}{6} & $\mathrm{E}_{1}$ & 0.0000 & 0.0000 & 0.0000 & 0.0011 & 0.7886 & 0.1558 & 0.0171 & 0.0347 & 5 \\
\hline & & $\mathrm{E}_{2}$ & 0.0000 & 0.0000 & 0.0000 & 0.0628 & 0.6611 & 0.0002 & 0.2332 & 0.0428 & 5 \\
\hline & & $\mathrm{E}_{3}$ & 0.0000 & 0.0000 & 0.0000 & 0.0003 & 0.0253 & 0.6916 & 0.2482 & 0.0346 & 6 \\
\hline & & $\mathrm{E}_{4}$ & 0.0000 & 0.0000 & 0.0000 & 0.0005 & 0.1416 & 0.6813 & 0.1354 & 0.0411 & 6 \\
\hline & & MIMD-S & 0.0000 & 0.0000 & 0.0000 & 0.0015 & 0.3840 & 0.5264 & 0.0923 & 0.0050 & 6 \\
\hline \multirow{2}{*}{85} & \multirow{2}{*}{4} & $\mathrm{E}_{4}$ & 0.0492 & 0.0000 & 0.0000 & 0.0139 & 0.0000 & 0.1727 & 0.7232 & 0.0411 & 7 \\
\hline & & MIMD-S & 0.0017 & 0.0001 & 0.0038 & 0.4988 & 0.0000 & 0.0122 & 0.4781 & 0.0045 & 4 \\
\hline
\end{tabular}

Table 5. Identification results.

\begin{tabular}{|c|c|c|c|c|}
\hline Methods & \multicolumn{2}{|c|}{ Training sample } & \multicolumn{2}{|c|}{ Testing sample } \\
\hline \multirow{8}{*}{ D-S } & $\mathrm{T}_{1}$ & 0.00 & $\mathrm{~T}_{1}$ & 0.00 \\
\hline & $\mathrm{T}_{2}$ & 0.25 & $\mathrm{~T}_{2}$ & 0.50 \\
\hline & $\mathrm{T}_{3}$ & 2.88 & $\mathrm{~T}_{3}$ & 1.75 \\
\hline & $\mathrm{T}_{5}$ & 1.88 & $\mathrm{~T}_{5}$ & 0.87 \\
\hline & $\mathrm{T}_{6}$ & 4.00 & $\mathrm{~T}_{6}$ & 1.38 \\
\hline & $\mathrm{T}_{7}$ & 0.62 & $\mathrm{~T}_{7}$ & 0.88 \\
\hline & Average & 1.96 & Average & 1.25 \\
\hline & Overall average & & 1.61 & \\
\hline \multirow{6}{*}{ PD-S } & $\mathrm{T}_{4}$ & 1.62 & $\mathrm{~T}_{4}$ & 1.63 \\
\hline & $\mathrm{T}_{5}$ & 0.87 & $\mathrm{~T}_{5}$ & 1.47 \\
\hline & $\mathrm{T}_{6}$ & 2.87 & $\mathrm{~T}_{6}$ & 1.38 \\
\hline & $\mathrm{T}_{7}$ & 0.50 & $\mathrm{~T}_{7}$ & 0 \\
\hline & Average & 1.07 & Average & 0.71 \\
\hline & Overall average & & 0.91 & \\
\hline \multirow{4}{*}{ MIMD-S } & $\mathrm{T}_{1}$ & 0.00 & $\mathrm{~T}_{1}$ & 0.00 \\
\hline & $\mathrm{T}_{2}$ & 0.53 & $\mathrm{~T}_{2}$ & 0.00 \\
\hline & $\mathrm{T}_{3}$ & 0.50 & $\mathrm{~T}_{3}$ & 0.13 \\
\hline & $\mathrm{T}_{4}$ & 2.10 & $\mathrm{~T}_{4}$ & 0.62 \\
\hline
\end{tabular}


Therefore, the average identification error rate of multiclassifier fusion is lower than that of a single classifier. The training sample identification error is $0.53 \%$, the testing sample identification error is $0.18 \%$ and the average identification error of the DBNMIMD-S are the lowest $0.36 \%$, as shown in Table 5 . The three identification error rates of DBNPD-S method are $1.07 \%, 0.71 \%$ and $0.91 \%$ respectively, which are higher than that of the DBNMIMD-S but lower than that of the DBN + D-S method. Actually, when the D-S evidence theory is applied to the multi-sensor fusion decision of the misalignment faults parameters identification, the identification framework determines that the identification results are obtained from independent evidence bodies. Therefore, the evidence conflict problem appears when the classification results are quite different, which makes the fusion identification results contradict with the reality. The PD-S evidence theory reduces the problem of evidence conflict to some extent, but the Pignistic method is only applicable to represent the distance within the Euclidian space, and it is difficult to accurately represent the similarity between evidence bodies. With the increase of evidence conflict, the average error rate of the PD-S evidence theory fusion decision increases. Because the mutual information measure has the characteristics of high matching accuracy and strong robustness, which is suitable for characterizing the similarity between the evidence bodies. Therefore, the DBN + MIND-S evidence theory has the lowest error rate of multi-sensor fusion decision.

\section{Conclusions}

Aiming at the problem of low identification rate of the traditional methods for dual-rotor misalignment fault quantitative identification, a dual-rotor misalignment identification method based on DBN and MIMD-S evidence theory was proposed. By comparing with the traditional methods, the following conclusions are drawn: - The improved D-S evidence theory based on the mutual information measure is effective. Compared with the traditional distance measure, the mutual information measure can better solve the problem of evidence conflict in the traditional D-S evidence theory.

- The DBNMIMD-S method proposed in this paper is suitable for the identification of misalignment fault parameters of dual-rotor. Compared with DBND-S and DBNPD-S, the DBNMIMD-S method proposed in this paper can reduce the error rate effectively.

\section{Conflicts of Interest}

The authors declare no conflict of interest.

This paper was supported by the National Natural Science Foundation of China (NO.11702091, NO.11672106), the Natural Science Foundation of Hunan Province of China under grant (No. 2018JJ3140, No.2019JJ50156) and the Excellent Youth Project of Hunan Education Department (No. 19B187).

\section{References}

[1] H. Zhang, X. Li, L. Jiang, D. Yang, A review of misalignment of aero-engine rotor system, Acta Aeronautica et Astronautica Sinica 40, 42-53 (2019)

[2] Q. Han, M. Wang, G. Zhao, G. Feng, A review of rotor systems with misalignment, Journal of Dynamics and Control 14, 1-13 (2016)

[3] M. Lal, R. Tiwari, Experimental estimation of misalignment effects in rotor-bearing-coupling systems, in: P. Pennacchi (Ed.), Proceedings of the 9th IFToMM International Conference on Rotor Dynamics, Springer, Cham 2015

[4] J. Rybczynski, The possibility of evaluating turbo-set bearing misalignment defects on the basis of bearing trajectory features, Mechanical Systems and Signal Processing 25, 521-536 (2011)

[5] P. Pennacchi, A.J.S. Vania, Vibration, Diagnosis and model based identification of a coupling misalignment, Sock and Vibration 12, 293-308 (2005)

[6] A.W. Lees, J.K. Sinha, M.I.J.M.S. Friswell, S. Processing, Model-based identification of rotating machines, Mechanical Systems \& Signal Processing 23, 1884-1893 (2009)

[7] N.H. Chandra, A.S. Sekhar, Fault detection in rotor bearing systems using time frequency techniques, Mechanical Systems and Signal Processing 72-73, 105-133 (2016)

[8] Y. Xia, J. Pang, L. Yang, Q. Zhao, X.J.A.A. Yang, Study on vibration response and orbits of misaligned rigid rotors connected by hexangular flexible coupling, Applied Acoustics 155, 286-296 (2019)

[9] H. Tejas, K. Patel, D.J.M. Ashish, Systems, S. Processing, Mechanical Systems \& Signal Processing 23, 2236-2252 (2009)

[10] T.H. Patel, A.K. Darpe, Vibration response of misaligned rotors, Journal of Sound and Vibration 325, 609-628 (2009)

[11] K. Schuhmann, K. Kirch, A. Knecht, M. Marszalek, F. Nez, J. Nuber, R. Pohl, I. Schulthess, L. Sinkunaite, M. Zeyen, A. Antognini, Passive alignment stability and auto-alignment of multipass amplifiers based on Fourier transforms, Applied Optics 58, 2904-2912 (2019)

[12] G. Szymanski, L. Wawrzyniuk, Effect of nonconstant mirror misalignment of moving mirror in Fourier transform spectrometer, Optical Engineering 54, 1-6 (2015)

[13] R. Yan, R.X. Gao, X. Chen, Wavelets for fault diagnosis of rotary machines: a review with applications, Signal Processing 96, 1-15 (2014)

[14] Y. Xiao, Y. Hong, X. Chen, W. Chen, The application of Dual-Tree Complex Wavelet Transform (DTCWT) energy entropy in misalignment fault Diagnosis of Doubly-Fed Wind Turbine (DFWT), Entropy 19, 1-14 (2017)

[15] A. Umbrajkaar, A. Krishnamoorthy, Vibration analysis using wavelet transform and fuzzy logic for shaft misalignment, Journal of Vibroengineering 20, 2855-2865 (2018)

[16] L. Yongjian, W. Hongjun, A revised Hilbert-Huang transform and its application to fault diagnosis in a rotor system, Sensors 18, 1-27 (2018)

[17] G. Dinardo, L. Fabbiano, G. Vacca, Energy-based indexes for analysis of vibrations from rotating machinery based on the Hilbert-Huang transform, in: E.P. Tomasini (Ed.), Proceedings of the 12th International AIVELA Conference on Vibration Measurements by Laser and Noncontact Techniques: Advances and Applications. (AIP Conference Proceedings) 2016

[18] X. Fu, H. Xu, Y. Lin, Z. Xiao, Vibration analysis on the mechanisms for hydropower unit rotors based on empirical 
mode decomposition, Journal of Optoelectronics and Advanced Materials 16, 689-696 (2014)

[19] S. Singh, N. Kumar, Combined rotor fault diagnosis in rotating machinery using empirical mode decomposition, Journal of Mechanical Science and Technology 28, 4869-4876 (2014)

[20] Z.R. Hou, Rolling bearing fault diagnosis based on wavelet packet and improved BP neural network for wind turbines, Applied Mechanics and Materials 347-350, 117-120 (2013)

[21] Y. Xue, Z. Li, B. Wang, Z. Zhao, F.J.A.I. Li, Nonlinear feature selection using Gaussian kernel SVM-RFE for fault diagnosis, Applied Intelligence 48, 3306-3331 (2018)

[22] N. Mayadevi, V.P. Mini, R.H. Kumar, S. Prins, Fuzzy-Based Intelligent Algorithm for Diagnosis of Drive Faults in Induction Motor Drive System, Arabian Journal for Science and Engineering 45, 1385-1395 (2020)

[23] Y. Dalian, M. Jingjing, Z. Fanyu, T. Jie, W. Guangbin, S. Yiping, Bearing fault diagnosis using a support vector machine optimized by an improved ant lion optimizer, Shock and Vibration 2019, 1-20 (2019)

[24] Y. Dalian, L. Yilun, L. Songbai, L. Xuejun, M. Liyong, Gear fault diagnosis based on support vector machine optimized by artificial bee colony algorithm, Mechanism and Machine Theory 90, 219-229 (2015)

[25] G.E. Hinton, R.R. Salakhutdinov, Reducing the dimensionality of data with neural networks, Science 313, 504-507 (2006)

[26] Z. Ling, X. Li, W. Zou, M. Liu, Joint haze-relevant features selection and transmission estimation via deep belief network for efficient single image dehazing, in: 2018 24th International Conference on Pattern Recognition (ICPR), IEEE, Beijing, China, 2018

[27] Z. Fengyu, Y. Jianqin, Y. Yang, Z. Haiting, Y. Xianfeng, Online recognition of human actions based on temporal deep belief neural network, Acta Automatica Snica 42, 1030-1039 (2016)

[28] M. Chen, J. Pan, Q. Zhao, Y. Yan, Multi-task learning in deep neural networks for Mandarin-English code-mixing speech recognition, IEICE Transactions on Information and Systems E99.D, 2554-2557 (2016)

[29] Z. Xiaoli, J. Minping, A new Local-Global Deep Neural Network and its application in rotating machinery fault diagnosis, Neurocomputing 366, 215-233 (2019)

[30] K. Zhao, H. Shao, Intelligent fault diagnosis of rolling bearing using adaptive deep gated recurrent unit, Neural Processing Letters 51, 1165-1184 (2020)

[31] X. He, J. Ma, Weak fault diagnosis of rolling bearing based on FRFT and DBN, Systems Science \& Control Engineering 8, 57-66 (2020)

[32] B. Han, X. Yang, Y. Ren, W. Lan, Comparisons of different deep learning-based methods on fault diagnosis for geared system, International Journal of Distributed Sensor Networks 15, 1-16 (2019)

[33] J. Li, X. Li, D. He, Y. Qu, Unsupervised rotating machinery fault diagnosis method based on integrated SAE-DBN and a binary processor, Journal of Intelligent Manufacturing 31, 1899-1916 (2020)

[34] P. Tamilselvan, P. Wang, Failure diagnosis using deep belief learning based health state classification, Reliability Engineering System Safety 115, 124-135 (2013)
[35] X. Jiang, Y. He, G. Li, Y. Liu, X.-P. Zhang, Building damage detection via superpixel-based belief fusion of space-borne SAR and optical images, IEEE Sensors Journal 20, 2008-2022 (2020)

[36] T. Tang, T. Hu, M. Chen, R. Lin, G. Chen, A deep convolutional neural network approach with information fusion for bearing fault diagnosis under different working conditions, Proceedings of the Institution of Mechanical Engineers Part C-Journal of Mechanical Engineering Science 203, 1989-1996 (2020)

[37] M.K. Abd Ghani, M.A. Mohammed, N. Arunkumar, S.A. Mostafa, D.A. Ibrahim, M.K. Abdullah, M.M. Jaber, E. Abdulhay, G. Ramirez-Gonzalez, M.A. Burhanuddin, Decision-level fusion scheme for nasopharyngeal carcinoma identification using machine learning techniques, Neural Computing \& Applications 32, 625-638 (2020)

[38] L.-x. Luo, Information fusion for wireless sensor network based on mass deep auto-encoder learning and adaptive weighted D-S evidence synthesis, Journal of Ambient Intelligence and Humanized Computing 11, 519-526 (2020)

[39] F. Xiao, A new divergence measure for belief functions in D-S evidence theory for multisensor data fusion, Information Sciences 514, 462-483 (2020)

[40] A. Stief, J.R. Ottewill, J. Baranowski, M. Orkisz, A PCA and two-stage bayesian sensor fusion approach for diagnosing electrical and mechanical faults in induction motors, IEEE Transactions on Industrial Electronics 66, 9510-9520 (2019)

[41] B. Tokhmechi, S. Ebrahimi, H. Azizi, S.R. Ghavami-Riabi, N. Farrokhi, Bayesian data fusion: a reliable approach for descriptive modeling of ore deposits, Journal of Mining and Environment 11, 63-76 (2020)

[42] D. Teekaraman, S. Sendhilkumar, G.S. Mahalakshmi, Semantic provenance based trustworthy users classification on book-based social network using fuzzy decision tree, International Journal of Uncertainty Fuzziness and Knowledge-Based Systems 28, 47-77 (2020)

[43] P. Bhattacharya, On the Dempster-Shafer evidence theory and non-hierarchical aggregation of belief structures, IEEE Transactions on Systems Man and Cybernetics Part A-Systems and Humans 30, 0-536 (2000)

[44] L.A. Zadeh, A simple view of the Dempster-Shafer theory of evidence and its implication for the rule of combination, AI Magazine 7, 85-90 (1986)

[45] A.L. Jousselme, D. Grenier, É. Bossé, A new distance between two bodies of evidence, Information Fusion 2, 91-101 (2001)

[46] L. Ma, F. Zhang, J. Chen, Synthetic rule of evidence based on pignistic probability distance, Computer Engineering and Applications 51, 61-66 (2015)

[47] J. Gonzalez-Lopez, S. Ventura, A. Cano, Distributed multilabel feature selection using individual mutual information measures, Knowledge-Based Systems 188, 1-10 (2020)

[48] L. Zheng, Using mutual information as a cocitation similarity measure, Scientometrics 119, 1695-1713 (2019)

[49] J. Tao, Y. Liu, D. Yang, Bearing fault diagnosis based on deep belief network and multisensor information fusion, Shock and Vibration 2016 (2016) 